\title{
Correction: Long non-coding RNA HoxA-AS3 interacts with EZH2 to regulate lineage commitment of mesenchymal stem cells
}

\author{
Xin-Xing Zhu ${ }^{1,2, *}$, Ya-Wei Yan ${ }^{1,2, *}$, Demeng Chen ${ }^{3}$, Chun-Zhi Ai ${ }^{1}$, Xifeng Lu ${ }^{4}$, Shan- \\ Shan $\mathrm{Xu}^{1}{ }^{1}$, Shan Jiang ${ }^{1}$, Gen-Shen Zhong ${ }^{5}$, Dong-Bao Chen ${ }^{6}$ and Yi-Zhou Jiang ${ }^{1}$ \\ ${ }^{1}$ Institute for Advanced Study, Shenzhen University, Shenzhen, Guangdong, China \\ ${ }^{2}$ Key Laboratory of Optoelectronic Devices and Systems of Ministry of Education and Guangdong, College of Optoelectronic \\ Engineering, Shenzhen University, Shenzhen, Guangdong, China \\ ${ }^{3}$ School of Dentistry, University of California, Los Angeles, CA, USA \\ ${ }^{4}$ Department of Physiology, Center for Diabetes, Obesity and Metabolism, Shenzhen University Health Science Center, \\ Shenzhen University, Shenzhen, Guangdong, China \\ ${ }^{5}$ Henan Key Laboratory of Neural Regeneration and Repairment, The First affiliated Hospital of Xinxiang Medical University, \\ Weihui, Henan, China \\ ${ }^{6}$ Department of Obstetrics and Gynecology, University of California, Irvine, CA, USA \\ * These authors contributed equally to this work
}

Published: April 03, 2018

Copyright: Zhu et al. This is an open-access article distributed under the terms of the Creative Commons Attribution License 3.0 (CC BY 3.0), which permits unrestricted use, distribution, and reproduction in any medium, provided the original author and source are credited.

This article has been corrected: The correct author affiliations are given below:

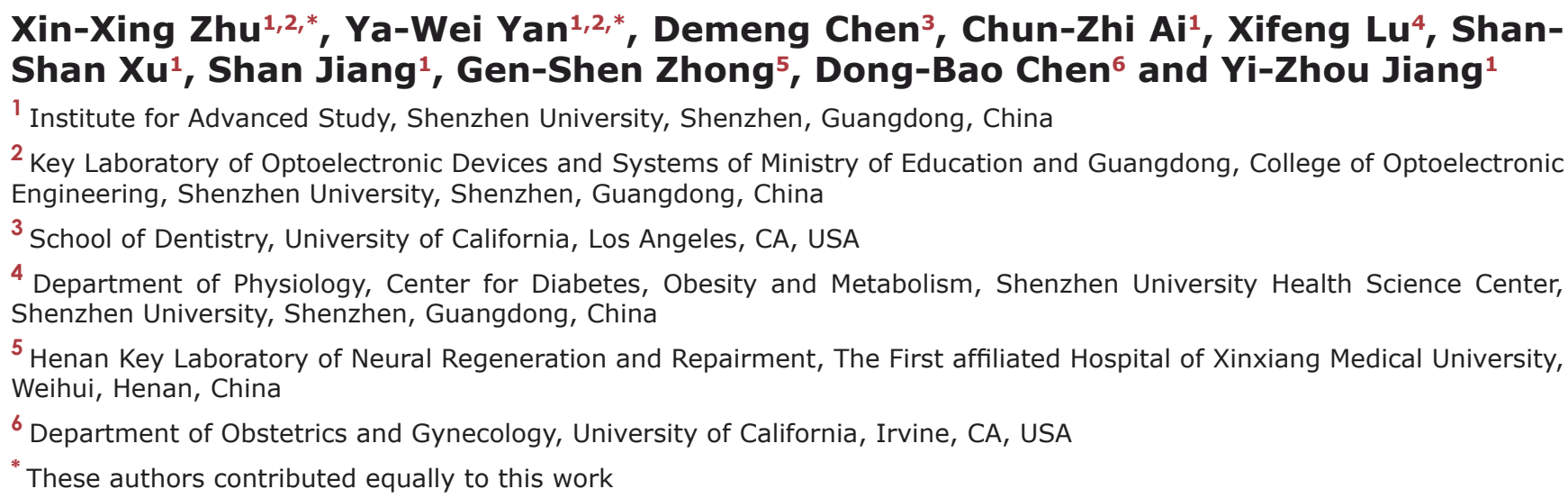
Shan $X^{1}{ }^{1}$, Shan Jiang ${ }^{1}$, Gen-Shen Zhong ${ }^{5}$, Dong-Bao Chen ${ }^{6}$ and Yi-Zhou Jiang ${ }^{1}$

${ }^{1}$ Institute for Advanced Study, Shenzhen University, Shenzhen, Guangdong, China

${ }^{2}$ Key Laboratory of Optoelectronic Devices and Systems of Ministry of Education and Guangdong, College of Optoelectronic Engineering, Shenzhen University, Shenzhen, Guangdong, China

${ }^{3}$ School of Dentistry, University of California, Los Angeles, CA, USA

${ }^{4}$ Department of Physiology, Center for Diabetes, Obesity and Metabolism, Shenzhen University Health Science Center, Shenzhen University, Shenzhen, Guangdong, China

${ }^{5}$ Henan Key Laboratory of Neural Regeneration and Repairment, The First affiliated Hospital of Xinxiang Medical University, Weihui, Henan, China

${ }^{6}$ Department of Obstetrics and Gynecology, University of California, Irvine, CA, USA

*These authors contributed equally to this work

Original article: Oncotarget. 2016; 7:63561-63570. https://doi.org/10.18632/oncotarget.11538 